Homology, Homotopy and Applications, vol.21(2), 2019, pp.377-394

\title{
HOMOTOPY CARTESIAN DIAGRAMS IN $n$-ANGULATED CATEGORIES
}

\author{
ZENGQIANG LIN AND YAN ZHENG \\ (communicated by Claude Cibils)
}

\begin{abstract}
It has been proved by Bergh and Thaule that the higher mapping cone axiom is equivalent to the higher octahedral axiom for $n$-angulated categories. In this paper we use homotopy cartesian diagrams to give several new equivalent statements of the higher mapping cone axiom. As an application we give a new and elementary proof of the fact that the stable category of a Frobenius $(n-2)$-exact category is an $n$-angulated category, which was first proved by Jasso.
\end{abstract}

\section{Introduction}

Let $n$ be an integer greater than or equal to three. The notion of $n$-angulated category was introduced by Geiss, Keller and Oppermann in [5] as the axiomatization of certain $(n-2)$-cluster tilting subcategories of triangulated categories. In particular, a 3 -angulated category is a classical triangulated category. Examples of $n$-angulated categories can be found in $[\mathbf{5}, \mathbf{4}, \mathbf{8}]$. Bergh and Thaule discussed the axioms of $n$ angulated categories in $[\mathbf{3}]$. They showed that for $n$-angulated categories the higher mapping cone axiom is equivalent to the higher octahedral axiom.

The first aim and motivation of this paper is to understand the higher octahedral axiom. The $n$-angle induced by the higher octahedral axiom is very mysterious because it involves a lot of objects and morphisms. How do these objects and morphisms behave together? What are the morphisms of $n$-angles hidden in the higher octahedral axiom?

The second motivation is to discuss other equivalent statements of the higher mapping cone axiom. As is well known, there are quite a few equivalent statements of octahedral axiom such as the homotopy cartesian axiom, the base change axiom, the cobase change axiom and so on, which are used to construct triangles under varied conditions. Do their higher versions exist?

It turns out that homotopy cartesian diagrams provide a useful method to achieve our two goals. The notion of homotopy cartesian diagrams in $n$-angulated categories was first introduced in [3]. For triangulated case, homotopy cartesian square can be found in $[\mathbf{1 0}, \mathbf{9}, \mathbf{7}]$. Since a homotopy cartesian square is the triangulated analogue

Received July 15, 2017, revised February 23, 2019; published on June 5, 2019.

2010 Mathematics Subject Classification: 18E30, 18E10.

Key words and phrases: $n$-angulated category, homotopy cartesian, mapping cone axiom, Frobenius $n$-exact category.

Article available at http://dx.doi.org/10.4310/HHA.2019.v21.n2.a21

Copyright (C) 2019, International Press. Permission to copy for private use granted. 
of pushout and pullback square in an abelian category, inspired by the definition of $(n-2)$-pushout and $(n-2)$-pullback diagrams in $(n-2)$-abelian categories [6], we give an equivalent definition of homotopy cartesian diagrams to avoid dealing with the signs $(-1)^{i}$ in the $n$-angle (see Remark 2.4 for details). Then we prove that the higher mapping cone axiom is equivalent to the higher homotopy cartesian axiom, which is implied but not state explicitly in [3]. Using homotopy cartesian diagrams, we will give several other equivalent statements of the higher mapping cone axiom.

It has been proved by Jasso that the stable category $\underline{\mathcal{M}}$ of a Frobenius $(n-2)$ exact category $(\mathcal{M}, \mathcal{X})$ is an $n$-angulated category [6, Theorem 5.11]. We will give an alternative proof of $[\mathbf{6}$, Theorem 5.11] by showing that the stable category $\mathcal{M}$ satisfies the higher homotopy cartesian axiom. Our proof seems more elementary since the standard $n$-angles in $\underline{\mathcal{M}}$ are naturally induced by the $(n-2)$-exact sequences in $\mathcal{X}$ and the homotopy cartesian diagrams are naturally induced by the $(n-2)$-pushout and $(n-2)$-pullback diagrams.

This paper is organized as follows. In Section 2 we first recall the definition of $n$ angulated categories, then introduce the notion of homotopy cartesian diagrams and provide some needed facts. In Section 3 we use homotopy cartesian diagrams to give several new equivalent statements of the higher mapping cone axiom; see Theorem 3.1, Corollary 3.4 and Corollary 3.6. In Section 4 we apply the new characterizations of the higher mapping cone axiom to give a new and elementary proof of the fact that the stable category of a Frobenius $(n-2)$-exact category is an $n$-angulated category; see Theorem 4.7.

\section{2. n-angulated categories and homotopy cartesian diagrams}

Throughout this paper, we always assume that $n$ is an integer greater than or equal to three. For convenience we recall the definition of $n$-angulated category from [5]. Let $\mathcal{C}$ be an additive category equipped with an automorphism $\Sigma: \mathcal{C} \rightarrow \mathcal{C}$. An $n$ - $\Sigma$-sequence in $\mathcal{C}$ is a sequence of morphisms

$$
X_{\bullet}=\left(X_{1} \stackrel{f_{1}}{\longrightarrow} X_{2} \stackrel{f_{2}}{\longrightarrow} X_{3} \stackrel{f_{3}}{\longrightarrow} \cdots \stackrel{f_{n-1}}{\longrightarrow} X_{n} \stackrel{f_{n}}{\longrightarrow} \Sigma X_{1}\right) .
$$

Its left rotation is the $n$ - $\sum$-sequence

$$
X_{2} \stackrel{f_{2}}{\longrightarrow} X_{3} \stackrel{f_{3}}{\longrightarrow} X_{4} \stackrel{f_{4}}{\longrightarrow} \cdots \stackrel{f_{n-1}}{\longrightarrow} X_{n} \stackrel{f_{n}}{\longrightarrow} \Sigma X_{1} \stackrel{(-1)^{n} \Sigma f_{1}}{\longrightarrow} \Sigma X_{2} .
$$

An $n$ - $\Sigma$-sequence $X$ • is exact if the induced sequence

$$
\cdots \rightarrow \mathcal{C}\left(-, X_{1}\right) \rightarrow \mathcal{C}\left(-, X_{2}\right) \rightarrow \cdots \rightarrow \mathcal{C}\left(-, X_{n}\right) \rightarrow \mathcal{C}\left(-, \Sigma X_{1}\right) \rightarrow \cdots
$$

is exact. A morphism of $n$ - $\Sigma$-sequences is a sequence of morphisms $\varphi_{\bullet}=\left(\varphi_{1}, \varphi_{2}, \ldots\right.$, $\left.\varphi_{n}\right)$ such that the following diagram

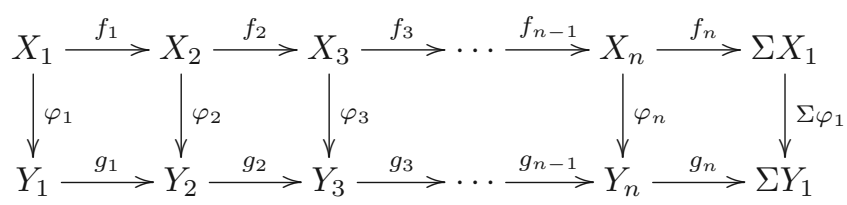


commutes, where each row is an $n$ - $\Sigma$-sequence. In this situation, we call $\varphi_{\bullet}$ a weak isomorphism if for some $1 \leqslant i \leqslant n$ both $\varphi_{i}$ and $\varphi_{i+1}\left(\right.$ with $\left.\varphi_{n+1}=\Sigma \varphi_{1}\right)$ are isomorphisms. We call $\varphi_{\bullet}$ an isomorphism if $\varphi_{1}, \varphi_{2}, \ldots, \varphi_{n}$ are all isomorphisms.

Definition $2.1([5])$. Let $\mathcal{C}$ be an additive category, $\Sigma$ an automorphism of $\mathcal{C}$ and $\Theta$ a collection of $n$ - $\Sigma$-sequences. We call $(\mathcal{C}, \Sigma, \Theta)$ a pre-n-angulated category and call the elements of $\Theta n$-angles if $\Theta$ satisfies the following axioms:

(N1) (a) $\Theta$ is closed under isomorphisms, direct sums and direct summands.

(b) For each object $X \in \mathcal{C}$ the trivial sequence

$$
(T X) \bullet=(X \stackrel{1}{\rightarrow} X \rightarrow 0 \rightarrow \cdots \rightarrow 0 \rightarrow \Sigma X)
$$

belongs to $\Theta$.

(c) For each morphism $f_{1}: X_{1} \rightarrow X_{2}$ in $\mathcal{C}$, there exists an $n$ - $\Sigma$-sequence in $\Theta$ whose first morphism is $f_{1}$.

(N2) An $n$ - $\sum$-sequence belongs to $\Theta$ if and only if its left rotation belongs to $\Theta$.

(N3) Each commutative diagram

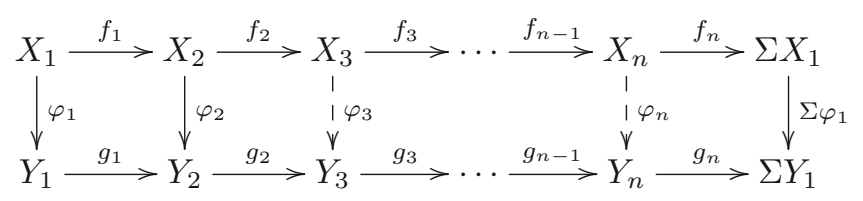

with rows in $\Theta$ can be completed to a morphism of $n$ - $\Sigma$-sequences.

If $\Theta$, moreover, satisfies the following axiom, then $(\mathcal{C}, \Sigma, \Theta)$ is called an $n$ angulated category:

(N4) In the situation of (N3), the morphisms $\varphi_{3}, \varphi_{4}, \ldots, \varphi_{n}$ can be chosen such that the mapping cone $C\left(\varphi_{\bullet}\right)$ :

$$
\begin{aligned}
X_{2} \oplus Y_{1} & \stackrel{\left(\begin{array}{ll}
-f_{2} & 0 \\
\varphi_{2} & g_{1}
\end{array}\right)}{\longrightarrow} X_{3} \oplus Y_{2} \stackrel{\left(\begin{array}{ll}
-f_{3} & 0 \\
\varphi_{3} & g_{2}
\end{array}\right)}{\longrightarrow} \cdots \\
& \stackrel{\left(\begin{array}{ll}
-f_{n} & 0 \\
\varphi_{n} & g_{n-1}
\end{array}\right)}{\longrightarrow} \Sigma X_{1} \oplus Y_{n} \stackrel{\left(\begin{array}{cc}
-\Sigma f_{1} & 0 \\
\Sigma \varphi_{1} & g_{n}
\end{array}\right)}{\longrightarrow} \Sigma X_{2} \oplus \Sigma Y_{1}
\end{aligned}
$$

belongs to $\Theta$.

The following theorem shows that for $n$-angulated categories the higher mapping cone axiom is equivalent to the higher octahedral axiom.

Theorem 2.2 ([3, Theorem 4.4]). Let $(\mathcal{C}, \Sigma, \Theta)$ be a pre-n-angulated category. Then $\Theta$ satisfies (N4) if and only if $\Theta$ satisfies $\left(N_{4}{ }^{*}\right)$ :

Given the following commutative diagram

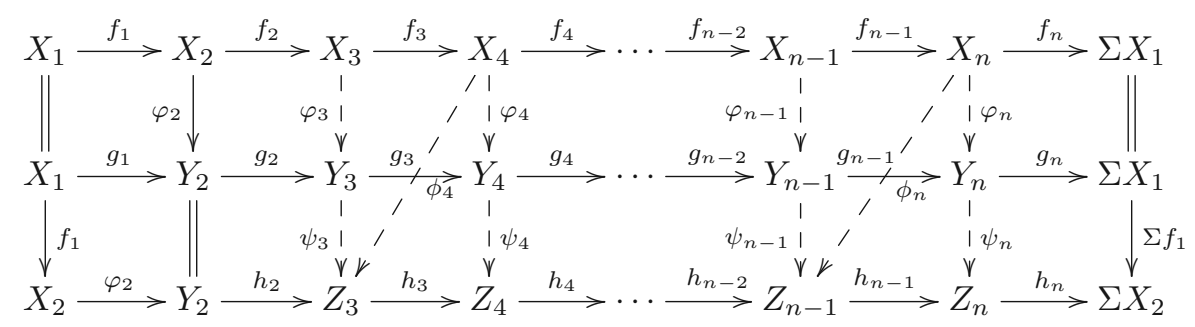


whose rows are $n$-angles, there exist morphisms $\varphi_{i}: X_{i} \rightarrow Y_{i}$ for $3 \leqslant i \leqslant n, \psi_{j}: Y_{j} \rightarrow$ $Z_{j}$ for $3 \leqslant j \leqslant n$ and $\phi_{k}: X_{k} \rightarrow Z_{k-1}$ for $4 \leqslant k \leqslant n$ such that each square in the above diagram commutes and the following $n$ - $\Sigma$-sequence

$$
\begin{aligned}
& X_{3} \stackrel{\left(\begin{array}{l}
f_{3} \\
\varphi_{3}
\end{array}\right)}{\longrightarrow} X_{4} \oplus Y_{3} \stackrel{\left(\begin{array}{cc}
-f_{4} & 0 \\
\varphi_{4} & -g_{3} \\
\phi_{4} & \psi_{3}
\end{array}\right)}{\longrightarrow} X_{5} \oplus Y_{4} \oplus Z_{3} \stackrel{\left(\begin{array}{ccc}
-f_{5} & 0 & 0 \\
-\varphi_{5} & -g_{4} & 0 \\
\phi_{5} & \psi_{4} & h_{3}
\end{array}\right)}{\longrightarrow} X_{6} \oplus Y_{5} \oplus Z_{4} \\
& \stackrel{\left(\begin{array}{ccc}
-f_{6} & 0 & 0 \\
\varphi_{6} & -g_{5} & 0 \\
\phi_{6} & \psi_{5} & h_{4}
\end{array}\right)}{\longrightarrow} \ldots \stackrel{\left(\begin{array}{ccc}
-f_{n-1} & 0 & 0 \\
(-1)^{n-1} \varphi_{n-1} & -g_{n-2} & 0 \\
\phi_{n-1} & \psi_{n-2} & h_{n-3}
\end{array}\right)}{\longrightarrow} X_{n} \oplus Y_{n-1} \oplus Z_{n-2} \\
& \stackrel{\left(\begin{array}{ccc}
(-1)^{n} \varphi_{n} & -g_{n-1} & 0 \\
\phi_{n} & \psi_{n-1} & h_{n-2}
\end{array}\right)}{\longrightarrow} Y_{n} \oplus Z_{n-1} \stackrel{\left(\psi_{n} h_{n-1}\right)}{\longrightarrow} Z_{n} \stackrel{\Sigma f_{2} \cdot h_{n}}{\longrightarrow} \Sigma X_{3}
\end{aligned}
$$

belongs to $\Theta$.

In the rest of this section we assume that all $n$-angles are in a pre- $n$-angulated category $(\mathcal{C}, \Sigma, \Theta)$.

Definition 2.3 (cf. [3]). The following commutative diagram

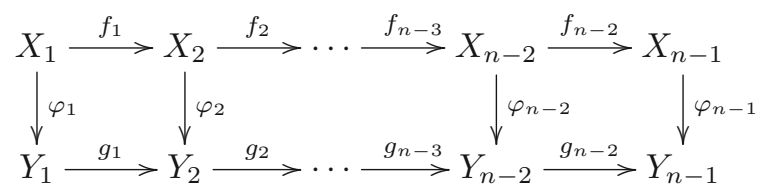

in a pre- $n$-angulated category $\mathcal{C}$ is called a homotopy cartesian diagram if the following $n$ - $\Sigma$-sequence

$$
\begin{aligned}
& X_{1} \stackrel{\left(\begin{array}{c}
-f_{1} \\
\varphi_{1}
\end{array}\right)}{\longrightarrow} X_{2} \oplus Y_{1} \stackrel{\left(\begin{array}{cc}
-f_{2} & 0 \\
\varphi_{2} & g_{1}
\end{array}\right)}{\longrightarrow} X_{3} \oplus Y_{2} \stackrel{\left(\begin{array}{ll}
-f_{3} & 0 \\
\varphi_{3} & g_{2}
\end{array}\right)}{\longrightarrow} \cdots \\
& \cdots \stackrel{\left(\begin{array}{ll}
-f_{n-2} & 0 \\
\varphi_{n-2} & g_{n-3}
\end{array}\right)}{\longrightarrow} X_{n-1} \oplus Y_{n-2} \stackrel{\left(\varphi_{n-1} g_{n-2}\right)}{\longrightarrow} Y_{n-1} \stackrel{\partial}{\longrightarrow} \Sigma X_{1}
\end{aligned}
$$

is an $n$-angle for some morphism $\partial: Y_{n-1} \rightarrow \Sigma X_{1}$, where $\partial$ is called a differential.

Remark 2.4. (a) The $n$-angle (2) in the definition of homotopy cartesian diagram is slightly different from the one in the definition given in $[\mathbf{3}]$. But the two $n$-angles are isomorphic:

$$
\begin{aligned}
& X_{1} \stackrel{\left(\begin{array}{c}
-f_{1} \\
\varphi_{1}
\end{array}\right)}{\longrightarrow} X_{2} \oplus Y_{1} \stackrel{\left(\begin{array}{cc}
-f_{2} & 0 \\
\varphi_{2} & g_{1}
\end{array}\right)}{\longrightarrow} X_{3} \oplus Y_{2} \stackrel{\left(\begin{array}{cc}
-f_{3} & 0 \\
\varphi_{3} & g_{2}
\end{array}\right)}{\longrightarrow} \cdots
\end{aligned}
$$

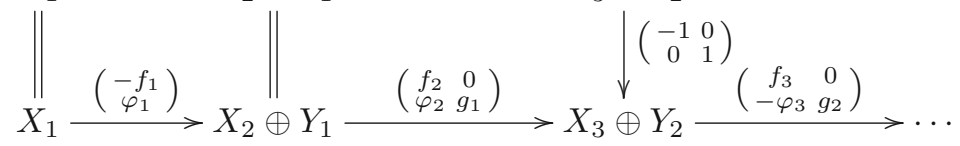

$$
\begin{aligned}
& \ldots \stackrel{\left(\begin{array}{cc}
-f_{n-2} & 0 \\
\varphi_{n-2} & g_{n-3}
\end{array}\right)}{\longrightarrow} X_{n-1} \oplus Y_{n-2} \stackrel{\left(\varphi_{n-1} g_{n-2}\right)}{\longrightarrow} Y_{n-1} \stackrel{\partial}{\longrightarrow} \Sigma X_{1} \\
& \ldots \stackrel{\left(\begin{array}{cc}
f_{n-2} & 0 \\
(-1)^{n} \varphi_{n-2} & g_{n-3}
\end{array}\right)}{\longrightarrow} X_{n-1} \underset{\left(\begin{array}{cc}
(-1)^{n+1} & 0 \\
0 & 1
\end{array}\right)}{ } \oplus Y_{n-2} \stackrel{\left((-1)^{n+1} \varphi_{n-1} g_{n-2}\right)}{\longrightarrow} Y_{n-1} \stackrel{\partial}{\longrightarrow} \Sigma X_{1}
\end{aligned}
$$


(b) Since a homotopy cartesian square is the triangulated analogue of a pullback and pushout square in an abelian category, we can compare our definition with the notion of an $(n-2)$-pushout and $(n-2)$-pullback diagram in an $(n-2)$-abelian category $[6]$.

Lemma 2.5 ([5, Lemma 2.4, Lemma 2.5]). Let $(\mathcal{C}, \Sigma, \Theta)$ be a pre-n-angulated category, then the following hold:

(a) All $n$-angles are exact.

(b) Let $\varphi_{\bullet}: X_{\bullet} \rightarrow Y_{\bullet}$ be a weak isomorphism of exact $n$ - $\Sigma$-sequences. Then $X_{\bullet}$ is an $n$-angle if and only if $Y_{\bullet}$ is an n-angle.

Lemma 2.6. Let

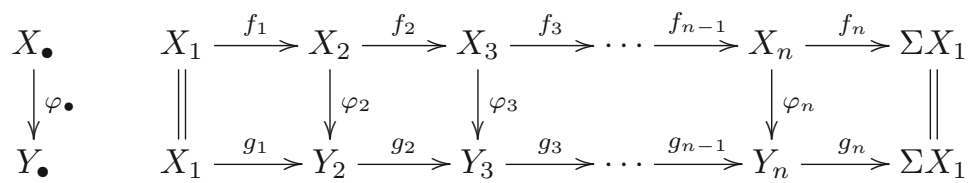

be a morphism of $n$-angles with $X_{1}=Y_{1}$. Then as an $n$ - $\Sigma$-sequence, the mapping cone $C\left(\varphi_{\bullet}\right)$ is isomorphic to $M\left(\varphi_{\bullet}\right) \oplus\left(X_{1}\right)_{\bullet}$, where

$$
\begin{aligned}
M\left(\varphi_{\bullet}\right) & =\left(X_{2} \stackrel{\left(\begin{array}{c}
-f_{2} \\
\varphi_{2}
\end{array}\right)}{\longrightarrow} X_{3} \oplus Y_{2} \stackrel{\left(\begin{array}{cc}
-f_{3} & 0 \\
\varphi_{3} & g_{2}
\end{array}\right)}{\longrightarrow} \cdots \stackrel{\left(\begin{array}{cc}
-f_{n-1} & 0 \\
\varphi_{n-1} & g_{n-2}
\end{array}\right)}{\longrightarrow} X_{n} \oplus Y_{n-1}\right. \\
& \left.\stackrel{\left(\varphi_{n} g_{n-1}\right)}{\longrightarrow} Y_{n} \stackrel{\Sigma f_{1} \cdot g_{n}}{\longrightarrow} \Sigma X_{2}\right), \quad\left(X_{1}\right) \bullet=\left(X_{1} \rightarrow 0 \rightarrow \cdots \rightarrow 0 \rightarrow \Sigma X_{1} \stackrel{1}{\rightarrow} \Sigma X_{1}\right) .
\end{aligned}
$$

In particular, $C\left(\varphi_{\bullet}\right)$ is exact if and only if $M\left(\varphi_{\bullet}\right)$ is exact.

Proof. It is easy to check the following isomorphism of $n$ - $\sum$-sequences:

$$
\begin{aligned}
& C\left(\varphi_{\bullet}\right): \quad X_{2} \oplus X_{1} \stackrel{\left(\begin{array}{cc}
-f_{2} & 0 \\
\varphi_{2} & g_{1}
\end{array}\right)}{\longrightarrow} X_{3} \oplus Y_{2} \stackrel{\left(\begin{array}{cc}
-f_{3} & 0 \\
\varphi_{3} & g_{2}
\end{array}\right)}{\longrightarrow} \cdots
\end{aligned}
$$

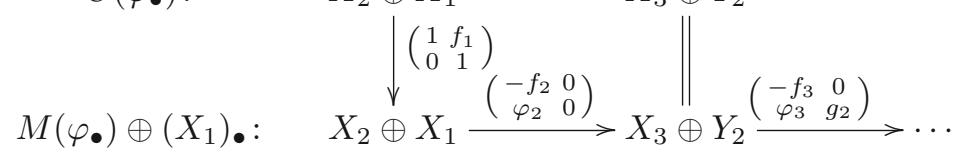

$$
\begin{aligned}
& \ldots \stackrel{\left(\begin{array}{cc}
-f_{n-1} & 0 \\
\varphi_{n-1} & g_{n-2}
\end{array}\right)}{\longrightarrow} X_{n} \oplus Y_{n-1} \stackrel{\left(\begin{array}{cc}
-f_{n} & 0 \\
\varphi_{n} & g_{n-1}
\end{array}\right)}{\longrightarrow} \Sigma X_{1} \oplus Y_{n} \stackrel{\left(\begin{array}{cc}
-\Sigma f_{1} & 0 \\
1 & g_{n}
\end{array}\right)}{\longrightarrow} \Sigma X_{2} \oplus \Sigma X_{1}
\end{aligned}
$$

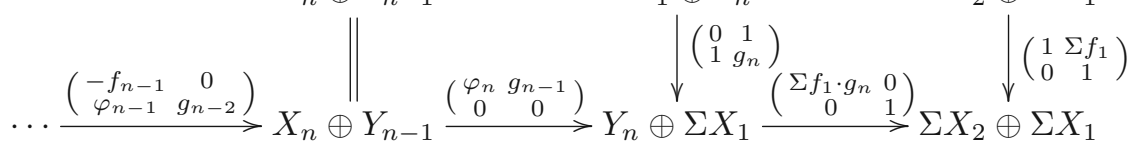

We note that $\left(X_{1}\right) \bullet$ is an $n$-angle since it is isomorphic to

$$
X_{1} \rightarrow 0 \rightarrow \cdots \rightarrow 0 \rightarrow \Sigma X_{1} \stackrel{(-1)^{n}}{\longrightarrow} \Sigma X_{1} .
$$

Thus $C\left(\varphi_{\bullet}\right)$ is exact if and only if $M\left(\varphi_{\bullet}\right)$ is exact. 
Lemma 2.7. Let
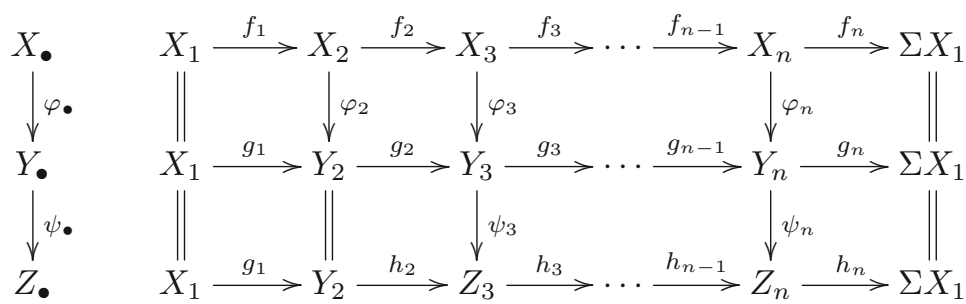

be a commutative diagram whose rows are $n$-angles. Then

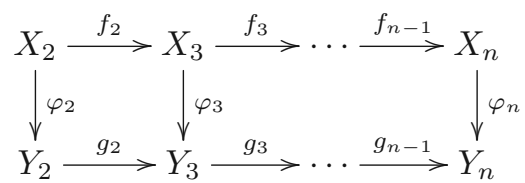

is a homotopy cartesian diagram with the differential $\Sigma f_{1} \cdot g_{n}$ if and only if

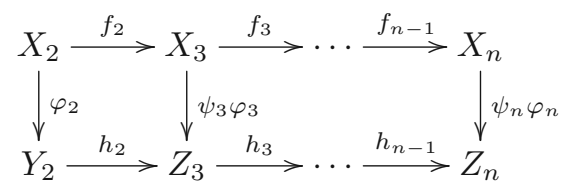

is a homotopy cartesian diagram with the differential $\Sigma f_{1} \cdot h_{n}$.

Proof. By Lemma 2.5(a), the $n$-angles $X_{\bullet}, Y_{\bullet}$ and $Z_{\bullet}$ are exact. Since the class of exact $n$ - $\Sigma$-sequences is closed under mapping cones, we obtain that the mapping cones $C\left(\varphi_{\bullet}\right)$ and $C\left(\psi_{\bullet} \varphi_{\bullet}\right)$ are also exact. It is easy to see that we have the following commutative diagram

$$
\begin{aligned}
& M\left(\varphi_{\bullet}\right): \quad X_{2} \stackrel{\left(\begin{array}{c}
-f_{2} \\
\varphi_{2}
\end{array}\right)}{\longrightarrow} X_{3} \oplus Y_{2} \stackrel{\left(\begin{array}{cc}
-f_{3} & 0 \\
\varphi_{3} & g_{2}
\end{array}\right)}{\longrightarrow} X_{4} \oplus Y_{3} \stackrel{\left(\begin{array}{cc}
-f_{4} & 0 \\
\varphi_{4} & g_{3}
\end{array}\right)}{\longrightarrow} \cdots \\
& M\left(\psi_{\bullet} \varphi_{\bullet}\right): \quad \|_{2} \stackrel{\left(\begin{array}{c}
-f_{2} \\
\varphi_{2}
\end{array}\right)}{\longrightarrow} X_{3} \oplus Y_{2} \stackrel{\left(\begin{array}{cc}
-f_{3} & 0 \\
\psi_{3} \varphi_{3} & h_{2}
\end{array}\right)}{\longrightarrow} X_{4} \stackrel{\downarrow}{\vartheta^{\oplus} Z_{3} \stackrel{\left(\begin{array}{ll}
1 & 0 \\
0 & \psi_{3}
\end{array}\right)}{\longrightarrow}\left(\begin{array}{cc}
-f_{4} & 0 \\
\psi_{4} \varphi_{4} & h_{3}
\end{array}\right)} \ldots \\
& \ldots \stackrel{\left(\begin{array}{cc}
-f_{n-1} & 0 \\
\varphi_{n-1} & g_{n-2}
\end{array}\right)}{\longrightarrow} X_{n} \oplus Y_{n-1} \stackrel{\left(\varphi_{n}, g_{n-1}\right)}{\longrightarrow} Y_{n} \stackrel{\Sigma f_{1} \cdot g_{n}}{\longrightarrow} \Sigma X_{2}
\end{aligned}
$$

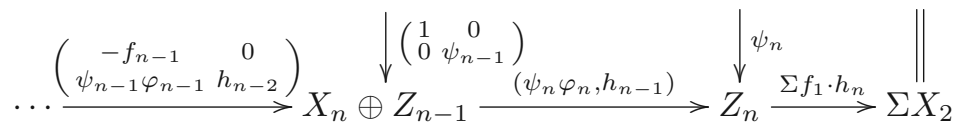

whose rows are exact $n$ - $\Sigma$-sequences by Lemma 2.6 . The result holds by definition and Lemma 2.5(b).

\section{Equivalent statements of higher mapping cone axiom}

In this section we provide some equivalent statements of higher mapping cone axiom to explain the higher octahedral axiom. We leave the dual statements to the reader. 
Theorem 3.1. Let $(\mathcal{C}, \Sigma, \Theta)$ be a pre-n-angulated category. Then $\Theta$ satisfies (N4) if and only if $\Theta$ satisfies (N4-1):

Given a commutative diagram

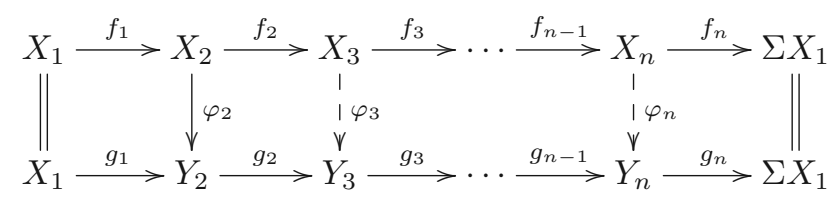

whose rows are $n$-angles, there exist morphisms $\varphi_{i}: X_{i} \rightarrow Y_{i}$ for $3 \leqslant i \leqslant n$ such that the above diagram is commutative and the following

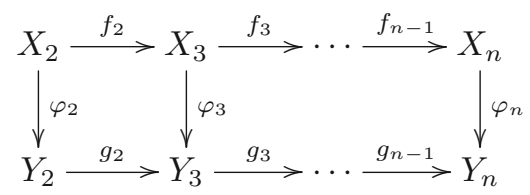

is a homotopy cartesian diagram whose differential is $\Sigma f_{1} \cdot g_{n}$.

Proof. Assume that $\Theta$ satisfies (N4), then there exist morphisms $\varphi_{i}: X_{i} \rightarrow Y_{i}$ for $3 \leqslant i \leqslant n$ such that the mapping cone $C\left(\varphi_{\bullet}\right)$ is an $n$-angle. Since the class of $n$ angles is closed under direct summands, the remaining part of (N4-1) follows from Lemma 2.6.

Conversely, we assume that $\Theta$ satisfies (N4-1). Given a commutative diagram

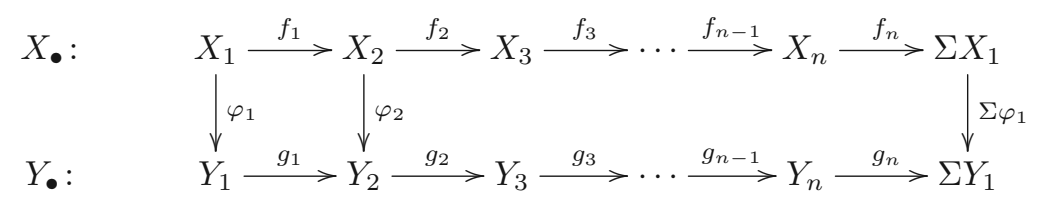

whose rows are $n$-angles. The following commutative diagram

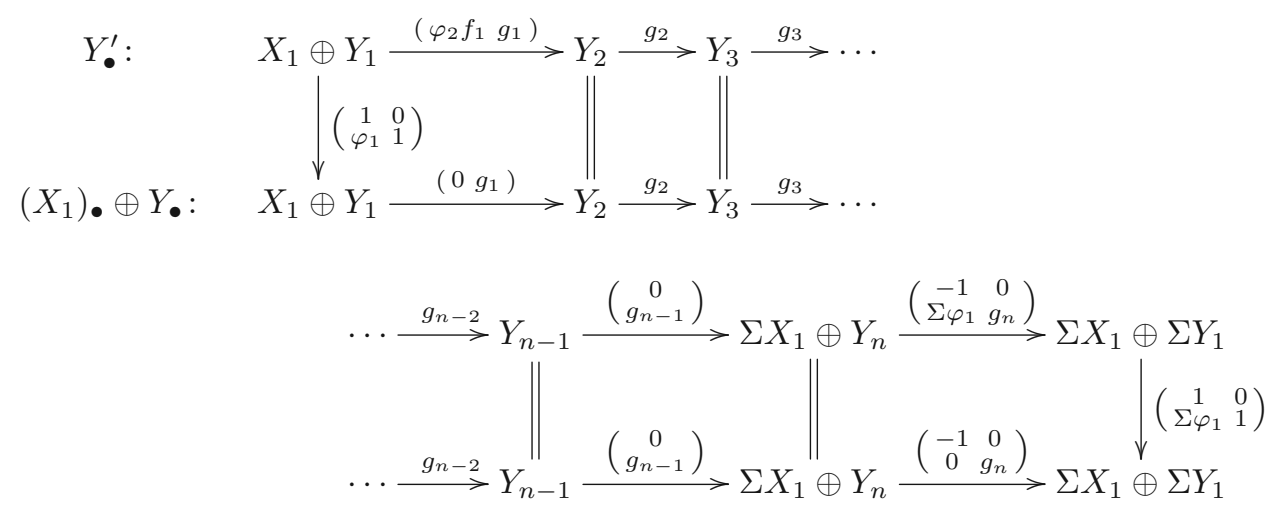

shows that $Y_{\bullet}^{\prime}$ is an $n$-angle since $\left(X_{1}\right) \bullet \oplus Y_{\bullet}$ is an $n$-angle. By (N4-1), the following 
diagram

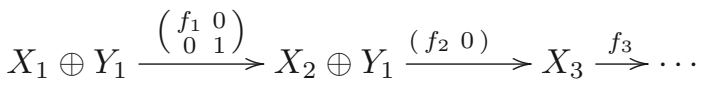

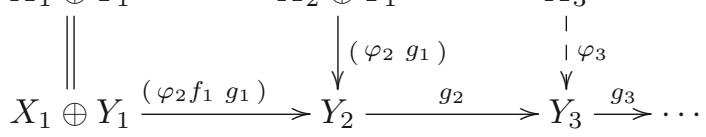

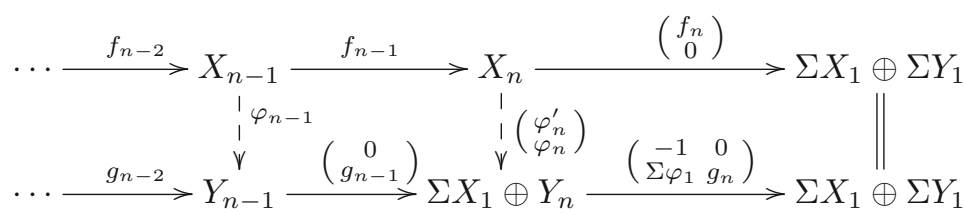

can be completed to a morphism of $n$-angles such that the following sequence

$$
\begin{aligned}
X_{2} \oplus Y_{1} & \stackrel{\left(\begin{array}{ll}
-f_{2} & 0 \\
\varphi_{2} & g_{1}
\end{array}\right)}{\longrightarrow} X_{3} \oplus Y_{2} \stackrel{\left(\begin{array}{ll}
-f_{3} & 0 \\
\varphi_{3} & g_{2}
\end{array}\right)}{\longrightarrow} X_{4} \oplus Y_{3} \stackrel{\left(\begin{array}{cc}
-f_{4} & 0 \\
\varphi_{4} & g_{3}
\end{array}\right)}{\longrightarrow} \cdots \\
& \ldots \stackrel{\left(\begin{array}{cc}
-f_{n-1} & 0 \\
\varphi_{n-1} & g_{n-2}
\end{array}\right)}{\longrightarrow} X_{n} \oplus Y_{n-1} \stackrel{\left(\begin{array}{cc}
\varphi_{n}^{\prime} & 0 \\
\varphi_{n} & g_{n-1}
\end{array}\right)}{\longrightarrow} \Sigma X_{1} \oplus Y_{n} \stackrel{\left(\begin{array}{cc}
-\Sigma f_{1} & 0 \\
\Sigma \varphi_{1} & g_{n}
\end{array}\right)}{\longrightarrow} \Sigma X_{2} \oplus \Sigma Y_{1}
\end{aligned}
$$

is an $n$-angle, where $\varphi_{n}^{\prime}=-f_{n}$ by the commutativity of the above rightmost square.

Since the proof of (N4-1) $\Rightarrow(\mathrm{N} 4)$ does not use (N3), we immediately obtain the following corollary.

Corollary 3.2. Let $\mathcal{C}$ be an additive category with an automorphism $\Sigma$ and $\Theta$ be a class of $n$ - $\Sigma$-sequences. Then $(\mathcal{C}, \Sigma, \Theta)$ is an n-angulated category if and only if $\Theta$ satisfies (N1), (N2) and (N4-1).

Remark 3.3. We can compare Corollary 3.2 with [1, Theorem 3.1], where the author proved that $(\mathcal{C}, \Sigma, \Theta)$ is an $n$-angulated category if and only if $\Theta$ satisfies (N1), (N2) and $\left(\mathrm{N} 4^{*}\right)$.

Corollary 3.4. Let $(\mathcal{C}, \Sigma, \Theta)$ be a pre-n-angulated category. Then the following statements are equivalent:

(a) $\Theta$ satisfies (N4).

(b) $\Theta$ satisfies (N4-2):

Given an $n$-angle $X_{1} \stackrel{f_{1}}{\longrightarrow} X_{2} \stackrel{f_{2}}{\longrightarrow} \cdots \stackrel{f_{n-1}}{\longrightarrow} X_{n} \stackrel{f_{n}}{\longrightarrow} \Sigma X_{1}$ and a morphism $\varphi_{1}: X_{1} \rightarrow$ $Y_{1}$, there exists a commutative diagram

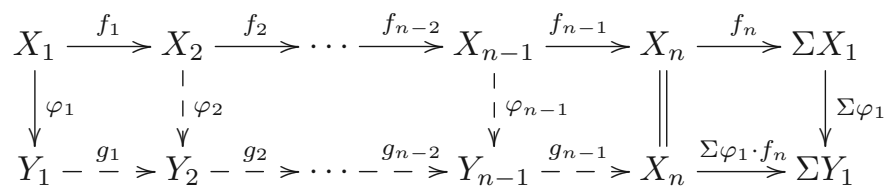


such that the second row is an n-angle and

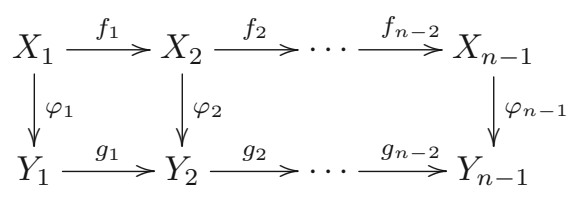

is a homotopy cartesian diagram where $(-1)^{n} f_{n} \cdot g_{n-1}$ is the differential.

(c) $\Theta$ satisfies (N4-3):

Given two morphisms $f_{1}: X_{1} \rightarrow X_{2}$ and $\varphi_{2}: X_{2} \rightarrow Y_{2}$, there exists a commutative diagram

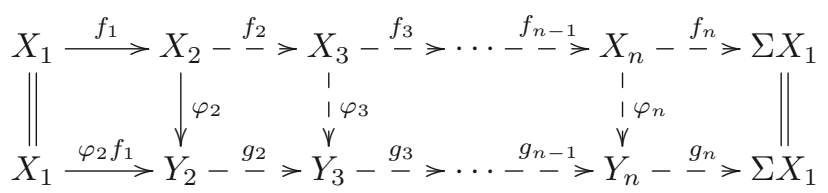

such that each row is an $n$-angle and

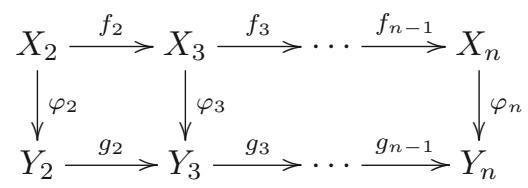

is a homotopy cartesian diagram where $\Sigma f_{1} \cdot g_{n}$ is the differential.

Proof. (a) $\Rightarrow$ (b). By (N2) and (N1)(c), we have the following commutative diagram

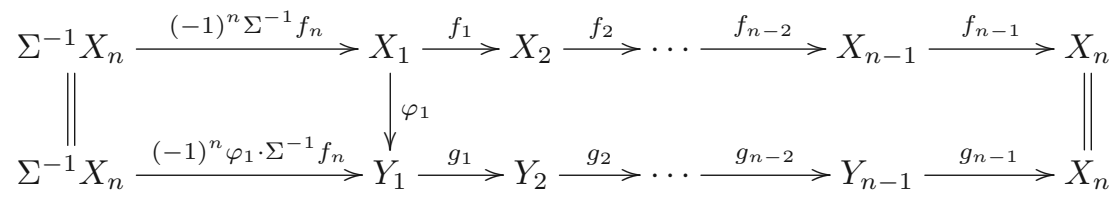

whose rows are $n$-angles. Now (b) follows from (N4-1) and Theorem 3.1.

(b) $\Rightarrow$ (c). For the morphism $f_{1}: X_{1} \rightarrow X_{2}$, by (N1)(c) and (N2) we assume that $X_{2} \stackrel{f_{2}}{\longrightarrow} \cdots \stackrel{f_{n-1}}{\longrightarrow} X_{n} \stackrel{f_{n}}{\longrightarrow} \Sigma X_{1} \stackrel{(-1)^{n} \Sigma f_{1}}{\longrightarrow} \Sigma X_{2}$ is an $n$-angle. Then (c) follows from (N4-2).

(c) $\Rightarrow$ (a). Given a commutative diagram

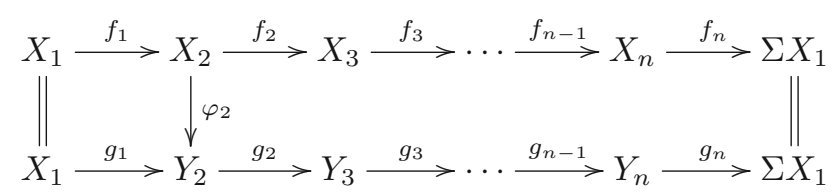

whose rows are $n$-angles. By (c), there exists a commutative diagram

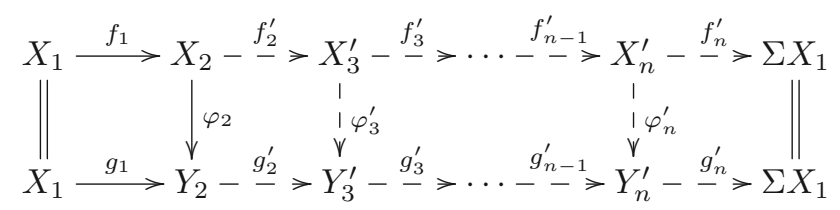


such that each row is an $n$-angle and

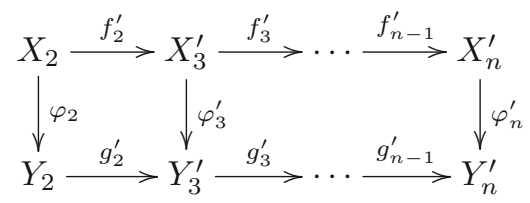

is a homotopy cartesian diagram where $\Sigma f_{1} \cdot g_{n}^{\prime}$ is the differential. It follows from (N3) that we have the following commutative diagram

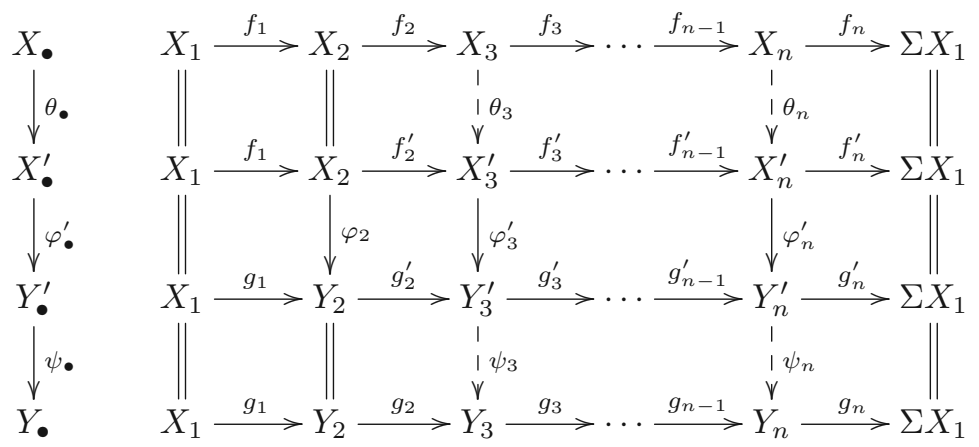

whose rows are $n$-angles. Lemma 2.7 and its dual imply that

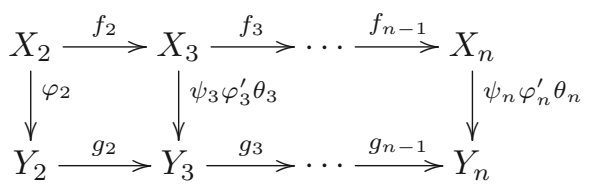

is a homotopy cartesian diagram and $\Sigma f_{1} \cdot g_{n}$ is the differential. Thus (N4-1) holds and (a) follows from Theorem 3.1.

Remark 3.5. The axiom (N4-1) and axiom (N4-2) are the higher analogues of homotopy cartesian axiom and cobase change axiom respectively. See $[\mathbf{9}, \mathbf{7}, \mathbf{2}]$ for reference.

Now we use the higher homotopy cartesian axiom (N4-1) to explain the higher octahedral axiom $\left(\mathrm{N} 4^{*}\right)$.

Corollary 3.6. Let $(\mathcal{C}, \Sigma, \Theta)$ be a pre-n-angulated category. Then the following statements are equivalent:

(a) $\Theta$ satisfies (N4).

(b) $\Theta$ satisfies (N4-4):

Given the following commutative diagram

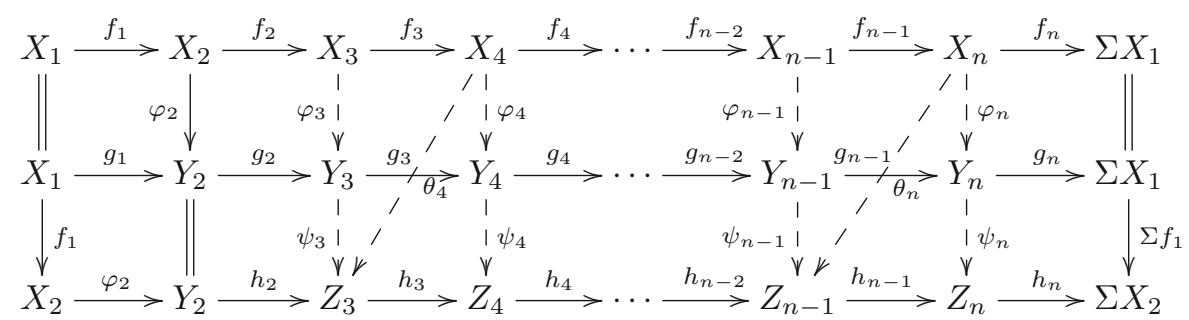


whose rows are $n$-angles, there exist morphisms $\varphi_{i}: X_{i} \rightarrow Y_{i}$ for $3 \leqslant i \leqslant n, \psi_{j}: Y_{j} \rightarrow$ $Z_{j}$ for $3 \leqslant j \leqslant n$ and $\theta_{k}: X_{k} \rightarrow Z_{k-1}$ for $4 \leqslant k \leqslant n$ such that the following diagram
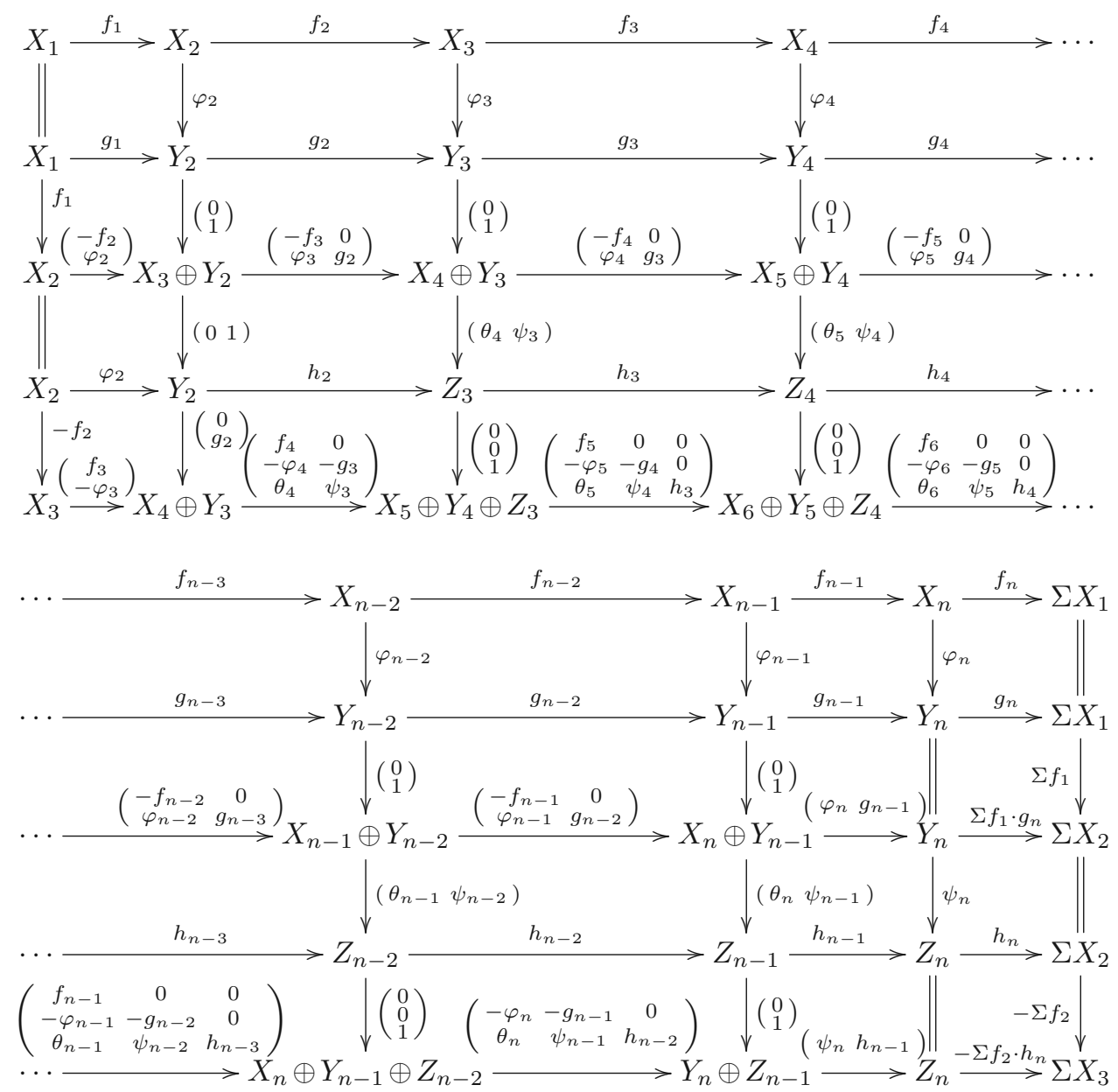

is commutative where each row is an n-angle.

(c) $\Theta$ satisfies (N4-5):

Given two morphisms $f_{1}: X_{1} \rightarrow X_{2}$ and $\varphi_{2}: X_{2} \rightarrow Y_{2}$, there exists a commutative diagram (3) such that each row is an n-angle.

Proof. (a) $\Rightarrow$ (b). By Theorem 3.1 and (N4-1), there exist morphisms $\varphi_{i}: X_{i} \rightarrow Y_{i}$ for $3 \leqslant i \leqslant n$ such that the diagram (3) involving the first two rows is commutative and the third row is the $n$-angle given by the homotopy cartesian diagram. By (N4-1) again, there exist morphisms $\psi_{j}: Y_{j} \rightarrow Z_{j}$ for $3 \leqslant j \leqslant n$ and $\theta_{k}: X_{k} \rightarrow Z_{k-1}$ for $4 \leqslant k \leqslant n$ such that the diagram involving the third row and the fourth row is commutative, and the $n$-angle given by the homotopy cartesian diagram is the direct sum of the fifth row and the trivial $n$-angle $\left(T Y_{2}\right)$ •. Other commutative squares are trivial.

(b) $\Rightarrow(\mathrm{c})$. It follows from $(\mathrm{N} 1)(\mathrm{c})$. 
(c) $\Rightarrow$ (a). The first three rows in diagram (3) implies that $\Theta$ satisfies (N4-3). Thus (a) follows from Corollary 3.4 .

Remark 3.7. If we replace $\theta_{k}$ by $(-1)^{k+1} \phi_{k}$ for $4 \leqslant k \leqslant n$ in the last row of diagram (3), then we have the following isomorphism of $n$-angles

$$
\begin{aligned}
& X_{3} \stackrel{\left(\begin{array}{c}
f_{3} \\
-\varphi_{3}
\end{array}\right)}{\longrightarrow} X_{4} \oplus Y_{3} \stackrel{\left(\begin{array}{cc}
f_{4} & 0 \\
-\varphi_{4} & -g_{3} \\
-\phi_{4} & \psi_{3}
\end{array}\right)}{\longrightarrow} X_{5} \oplus Y_{4} \oplus Z_{3} \stackrel{\left(\begin{array}{ccc}
f_{5} & 0 & 0 \\
-\varphi_{5} & -g_{4} & 0 \\
\phi_{5} & \psi_{4} & h_{3}
\end{array}\right)}{\longrightarrow} X_{6} \oplus Y_{5} \oplus Z_{4} \stackrel{\left(\begin{array}{ccc}
f_{6} & 0 & 0 \\
-\varphi_{6} & -g_{5} & 0 \\
-\phi_{6} & \psi_{5} & h_{4}
\end{array}\right)}{\longrightarrow} \ldots
\end{aligned}
$$

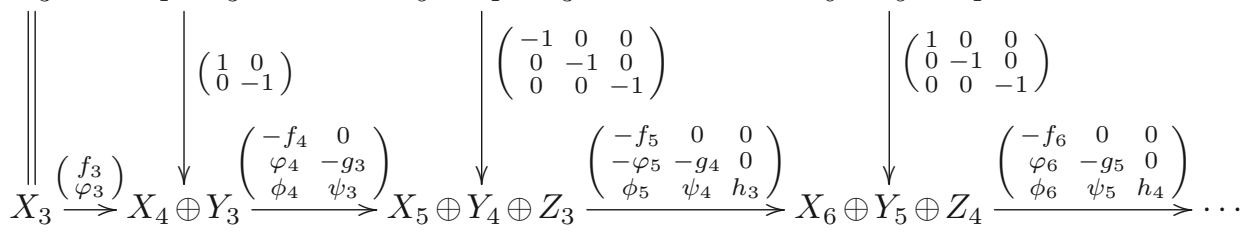

$$
\begin{aligned}
& \begin{array}{c}
\left(\begin{array}{ccc}
f_{n-1} & 0 & 0 \\
-\varphi_{n-1} & -g_{n-2} & 0 \\
(-1)^{n} \phi_{n-1} & \psi_{n-2} & h_{n-3}
\end{array}\right) \quad\left(\begin{array}{ccc}
-\varphi_{n} & -g_{n-1} & 0 \\
(-1)^{n+1} \phi_{n} & \psi_{n-1} & h_{n-2}
\end{array}\right) \quad \begin{array}{c}
\left(\psi_{n} h_{n-1}\right)-\Sigma f_{2} \cdot h_{n} \\
\longrightarrow
\end{array} X_{n}^{\longrightarrow} \oplus Y_{n-1} \oplus Z_{n-2} \stackrel{Z_{n}}{\longrightarrow} Y_{n} \oplus Z_{n-1} \longrightarrow Z_{n} \longrightarrow
\end{array} \\
& \begin{array}{c}
\left(\begin{array}{ccc}
-f_{n-1} & 0 & 0 \\
(-1)^{n-1} \varphi_{n-1} & -g_{n-2} & 0 \\
\phi_{n-1} & \psi_{n-2} & h_{n-3}
\end{array}\right) \\
\cdots
\end{array}
\end{aligned}
$$

where the second row is the $n$-angle (1) given by the higher octahedral axiom $\left(\mathrm{N} 4^{*}\right)$. Indeed, (N4-4) is nothing but the proof of (N4) implying (N4*). Moreover, by (N4-4) we easily obtain the morphisms of $n$-angles hidden in $\left(\mathrm{N} 4^{*}\right)$.

\section{An application}

In this section we apply the idea of homotopy cartesian diagram developed in Section 3 to give a new proof of [6, Theorem 5.11]. We first recall some basic definitions and facts on Frobenius $n$-exact categories from [6].

Definition 4.1. Let $\mathcal{C}$ be an additive category and $f_{1}: X_{1} \rightarrow X_{2}$ be a morphism in $\mathcal{C}$. An $(n-2)$-cokernel of $f_{1}$ is a sequence

$$
\left(f_{2}, f_{3}, \ldots, f_{n-1}\right): X_{2} \stackrel{f_{2}}{\longrightarrow} X_{3} \stackrel{f_{3}}{\longrightarrow} \cdots \stackrel{f_{n-1}}{\longrightarrow} X_{n}
$$

such that the induced sequence

$$
0 \rightarrow \mathcal{C}\left(X_{n}, Y\right) \rightarrow \mathcal{C}\left(X_{n-1}, Y\right) \rightarrow \cdots \rightarrow \mathcal{C}\left(X_{3}, Y\right) \rightarrow \mathcal{C}\left(X_{2}, Y\right) \rightarrow \mathcal{C}\left(X_{1}, Y\right)
$$

is exact for each $Y \in \mathcal{C}$. In this case, the sequence

$$
X_{1} \stackrel{f_{1}}{\longrightarrow} X_{2} \stackrel{f_{2}}{\longrightarrow} \cdots \stackrel{f_{n-2}}{\longrightarrow} X_{n-1} \stackrel{f_{n-1}}{\longrightarrow} X_{n} \rightarrow 0
$$

is called right $(n-2)$-exact. The notion of $(n-2)$-kernel and of left $(n-2)$-exact sequence are defined dually. A sequence

$$
0 \rightarrow X_{1} \stackrel{f_{1}}{\longrightarrow} X_{2} \stackrel{f_{2}}{\longrightarrow} \cdots \stackrel{f_{n-2}}{\longrightarrow} X_{n-1} \stackrel{f_{n-1}}{\longrightarrow} X_{n} \rightarrow 0
$$

is called $(n-2)$-exact if it is both right and left $(n-2)$-exact. 
Definition 4.2. A commutative diagram

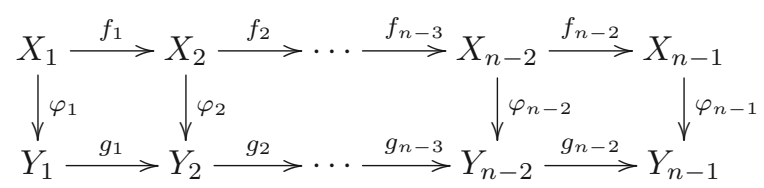

is an $(n-2)$-pushout diagram if the following sequence

$$
\begin{aligned}
X_{1} \stackrel{\left(\begin{array}{c}
-f_{1} \\
\varphi_{1}
\end{array}\right)}{\longrightarrow} X_{2} \oplus Y_{1} \stackrel{\left(\begin{array}{cc}
-f_{2} & 0 \\
\varphi_{2} & g_{1}
\end{array}\right)}{\longrightarrow} X_{3} \oplus Y_{2} \stackrel{\left(\begin{array}{ll}
-f_{3} & 0 \\
\varphi_{3} & g_{2}
\end{array}\right)}{\longrightarrow} \cdots \\
\ldots \stackrel{\left(\begin{array}{cc}
-f_{n-2} & 0 \\
\varphi_{n-2} & g_{n-3}
\end{array}\right)}{\longrightarrow} X_{n-1} \oplus Y_{n-2} \stackrel{\left(\varphi_{n-1} g_{n-2}\right)}{\longrightarrow} Y_{n-1} \rightarrow 0
\end{aligned}
$$

is right $(n-2)$-exact.

Let $\mathcal{M}$ be an additive category and $\mathcal{X}$ be a class of $(n-2)$-exact sequences in $\mathcal{M}$. The pair $(\mathcal{M}, \mathcal{X})$ is called an $(n-2)$-exact category if it satisfies some axioms which are similar to those of exact categories (see [6, Definition 4.2] for details). We will frequently use the following lemma and its dual.

Lemma 4.3 ([6, Proposition 4.8]). Let $(\mathcal{M}, \mathcal{X})$ be an $(n-2)$-exact category. If the sequence

$$
0 \rightarrow X_{1} \stackrel{f_{1}}{\longrightarrow} X_{2} \stackrel{f_{2}}{\longrightarrow} \cdots \stackrel{f_{n-2}}{\longrightarrow} X_{n-1} \stackrel{f_{n-1}}{\longrightarrow} X_{n} \rightarrow 0
$$

is an $(n-2)$-exact sequence in $\mathcal{X}$, then the following statements are equivalent:

(a) The diagram

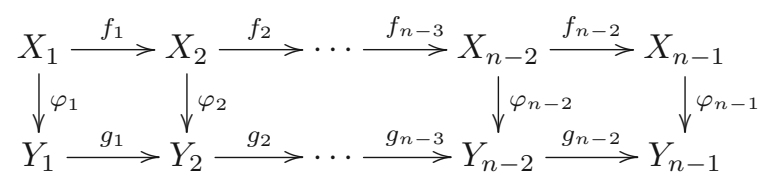

is both an $(n-2)$-pushout and an $(n-2)$-pullback diagram.

(b) The sequence

$$
\begin{aligned}
0 \rightarrow X_{1} \stackrel{\left(\begin{array}{l}
-f_{1} \\
\varphi_{1}
\end{array}\right)}{\longrightarrow} X_{2} \oplus Y_{1} \stackrel{\left(\begin{array}{ll}
-f_{2} & 0 \\
\varphi_{2} & g_{1}
\end{array}\right)}{\longrightarrow} X_{3} \oplus Y_{2} \stackrel{\left(\begin{array}{ll}
-f_{3} & 0 \\
\varphi_{3} & g_{2}
\end{array}\right)}{\longrightarrow} \cdots \\
\cdots \\
\cdots \stackrel{\left(\begin{array}{cc}
-f_{n} & 0 \\
\varphi_{n-2} & g_{n-3}
\end{array}\right)}{\longrightarrow} X_{n-1} \oplus Y_{n-2} \stackrel{\left(\varphi_{n-1} g_{n-2}\right)}{\longrightarrow} Y_{n-1} \rightarrow 0
\end{aligned}
$$

is an $(n-2)$-exact sequence in $\mathcal{X}$.

(c) There exists a commutative diagram

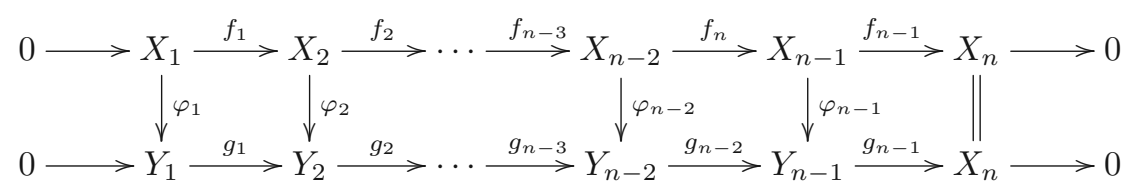

whose rows are $(n-2)$-exact sequences in $\mathcal{X}$. 
Let $(\mathcal{M}, \mathcal{X})$ be an $(n-2)$-exact category. Recall that an object $I \in \mathcal{M}$ is called $\mathcal{X}$-injective if for any $(n-2)$-exact sequence

$$
0 \rightarrow X_{1} \stackrel{f_{1}}{\longrightarrow} X_{2} \stackrel{f_{2}}{\longrightarrow} \cdots \stackrel{f_{n-2}}{\longrightarrow} X_{n-1} \stackrel{f_{n-1}}{\longrightarrow} X_{n} \rightarrow 0
$$

in $\mathcal{X}$ and any morphism $g \in \mathcal{M}\left(X_{1}, I\right)$, there is a morphism $h \in \mathcal{M}\left(X_{2}, I\right)$ such that $g=h f_{1}$. We denote by $\mathcal{I}$ the full subcategory of $\mathcal{M}$ consisting of $\mathcal{X}$-injectives. We say $(\mathcal{M}, \mathcal{X})$ has enough $\mathcal{X}$-injectives if for any object $X \in \mathcal{M}$, there is an $(n-2)$-exact sequence

$$
0 \rightarrow X \stackrel{i_{1}}{\longrightarrow} I_{1}(X) \stackrel{i_{2}}{\longrightarrow} I_{2}(X) \rightarrow \cdots \stackrel{i_{n-2}}{\longrightarrow} I_{n-2}(X) \stackrel{i_{n-1}}{\longrightarrow} Y \rightarrow 0
$$

in $\mathcal{X}$ such that $I_{i}(X) \in \mathcal{I}$. We say $(\mathcal{M}, \mathcal{X})$ is Frobenius if it has enough $\mathcal{X}$-injectives, enough $\mathcal{X}$-projectives and if $\mathcal{X}$-injective objects and $\mathcal{X}$-projective objects coincide.

From now on, we assume that $(\mathcal{M}, \mathcal{X})$ is a Frobenius $(n-2)$-exact category. We denote by $\underline{\mathcal{M}}$ the stable category $\mathcal{M} /[\mathcal{I}]$. Given a morphism $f: X \rightarrow Y$ in $\mathcal{M}$, we denote by $f$ the image of $f$ in $\underline{\mathcal{M}}$ under the canonical functor $\mathcal{M} \rightarrow \underline{\mathcal{M}}$.

The following lemma is clear. It is a variant of [6, Lemma 2.1].

Lemma 4.4. Assume that we have the following two commutative diagrams

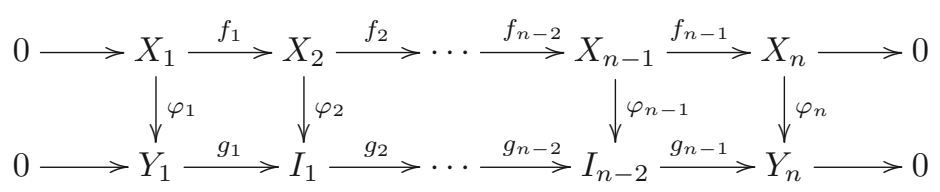

and

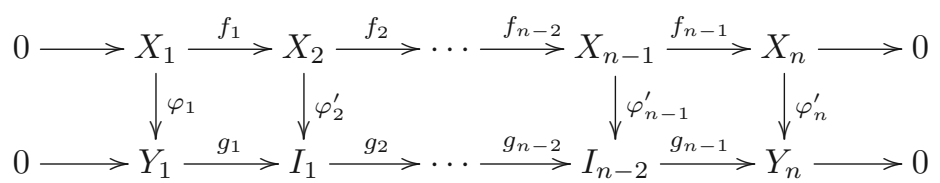

whose rows are $(n-2)$-exact sequences in $\mathcal{X}$ and $I_{i} \in \mathcal{I}$. Then $\underline{\varphi_{n}}=\underline{\varphi_{n}^{\prime}}$ in $\underline{\mathcal{M}}$.

Following [6, Proposition 5.8], there exists an automorphism $\Sigma: \underline{\mathcal{M}} \rightarrow \underline{\mathcal{M}}$. For convenience we recall the definition of $\Sigma$. For each $X \in \mathcal{M}$, we choose an $(n-2)$ exact sequence

$$
0 \rightarrow X \stackrel{i_{1}}{\longrightarrow} I_{1}(X) \stackrel{i_{2}}{\longrightarrow} I_{2}(X) \rightarrow \cdots \stackrel{i_{n-2}}{\longrightarrow} I_{n-2}(X) \stackrel{i_{n-1}}{\longrightarrow} \Sigma X \rightarrow 0
$$

in $\mathcal{X}$ such that $I_{i}(X) \in \mathcal{I}$. For each morphism $f: X \rightarrow Y$, we have the following commutative diagram

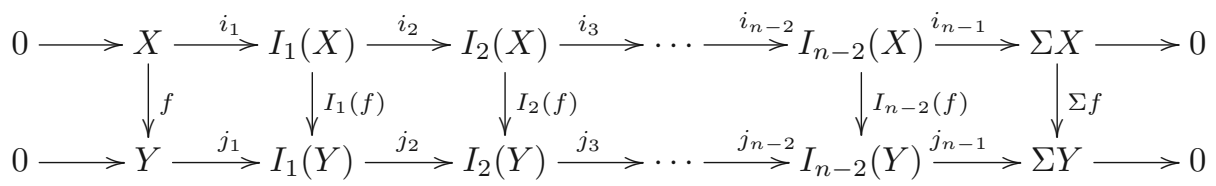

with rows in $\mathcal{X}$. By Lemma 4.4 the morphism $\Sigma f$ does not depend on the choice of $I_{i}(f)$. We define $\Sigma \underline{f}=\Sigma \underline{\Sigma f}$. It is easily seen that the functor $\Sigma$ is an automorphism of $\underline{\mathcal{M}}$. 
Given an $(n-2)$-exact sequence

$$
0 \rightarrow X_{1} \stackrel{f_{1}}{\longrightarrow} X_{2} \stackrel{f_{2}}{\longrightarrow} \cdots \stackrel{f_{n-2}}{\longrightarrow} X_{n-1} \stackrel{f_{n-1}}{\longrightarrow} X_{n} \rightarrow 0
$$

in $\mathcal{X}$, we call the sequence

$$
X_{1} \stackrel{f_{1}}{\longrightarrow} X_{2} \stackrel{f_{2}}{\longrightarrow} \cdots \stackrel{f_{n-2}}{\longrightarrow} X_{n-1} \stackrel{f_{n-1}}{\longrightarrow} X_{n} \stackrel{(-1)^{n}}{\stackrel{f_{n}}{\longrightarrow}} \Sigma X_{1}
$$

a standard $n$-angle if there exists the following commutative diagram

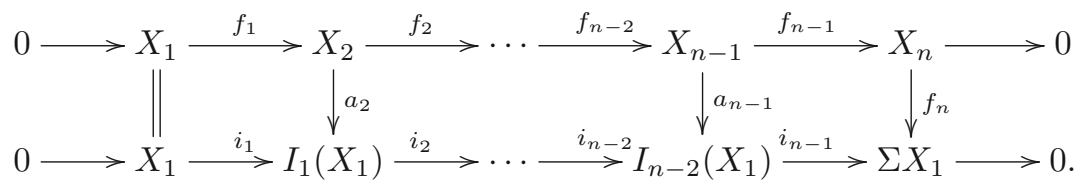

We denote by $\Theta(\mathcal{X})$ the class of $n$ - $\Sigma$-sequences which are isomorphic to standard $n$-angles.

Lemma 4.5. Let $(\mathcal{M}, \mathcal{X})$ be a Frobenius $(n-2)$-exact category. Assume that the following is a commutative diagram

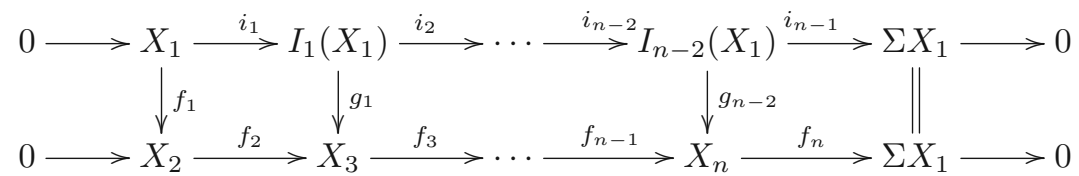

whose rows are $(n-2)$-exact sequences in $\mathcal{X}$ and $I_{i}\left(X_{1}\right) \in \mathcal{I}$. Then the sequence

$$
X_{1} \stackrel{f_{1}}{\longrightarrow} X_{2} \stackrel{f_{2}}{\longrightarrow} X_{3} \stackrel{f_{3}}{\longrightarrow} \cdots \stackrel{f_{n-1}}{\longrightarrow} X_{n} \stackrel{f_{n}}{\longrightarrow} \Sigma X_{1}
$$

belongs to $\Theta(\mathcal{X})$.

Proof. By Lemma 4.3 we have the following morphism of $(n-2)$-exact sequences

$$
\begin{aligned}
& 0 \longrightarrow X_{1} \stackrel{\left(\begin{array}{c}
-i_{1} \\
f_{1}
\end{array}\right)}{\longrightarrow} I_{1}\left(X_{1}\right) \oplus X_{2} \stackrel{\left(\begin{array}{cc}
-i_{2} & 0 \\
g_{1} & f_{2}
\end{array}\right)}{\longrightarrow} I_{2}\left(X_{1}\right) \oplus X_{3} \stackrel{\left(\begin{array}{cc}
-i_{3} & 0 \\
g_{2} & f_{3}
\end{array}\right)}{\longrightarrow} \ldots
\end{aligned}
$$

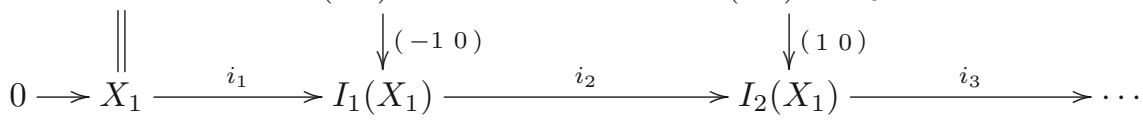

$$
\begin{aligned}
& \ldots \stackrel{\left(\begin{array}{cc}
-i_{n-2} & 0 \\
g_{n-3} & f_{n-2}
\end{array}\right)}{\longrightarrow} I_{n-2}\left(X_{1}\right) \oplus X_{n-1} \stackrel{\left(g_{n-2}, f_{n-1}\right)}{\longrightarrow} X_{n} \longrightarrow 0 \\
& \downarrow\left((-1)^{n-2} 0\right) i_{n_{n-1}} \quad \downarrow \downarrow(-1)^{n-2} f_{n}
\end{aligned}
$$

Thus the sequence

$$
X_{1} \stackrel{f_{1}}{\longrightarrow} X_{2} \stackrel{f_{2}}{\longrightarrow} X_{3} \stackrel{\stackrel{f_{3}}{\longrightarrow}}{\longrightarrow} \stackrel{\stackrel{f_{n-1}}{\longrightarrow}}{\longrightarrow} X_{n} \stackrel{f_{n}}{\longrightarrow} \Sigma X_{1}
$$

belongs to $\Theta(\mathcal{X})$ by definition.

Remark 4.6. We point out that our definition of standard $n$-angles is different from the definition given in [6]. They are the same up to isomorphisms by Lemma 4.5 and [6, Lemma 5.10]. 
The following theorem was first proved by Jasso. We will give a new proof.

Theorem 4.7 ([6, Theorem 5.11]). Let $(\mathcal{M}, \mathcal{X})$ be a Frobenius $(n-2)$-exact category, then $(\underline{\mathcal{M}}, \Sigma, \Theta(\mathcal{X}))$ is an n-angulated category.

Proof. By Corollary 3.2, we only need to show that $\Theta(\mathcal{X})$ satisfies (N1), (N2) and (N4-1). Here we only prove (N4-1).

Given a commutative diagram

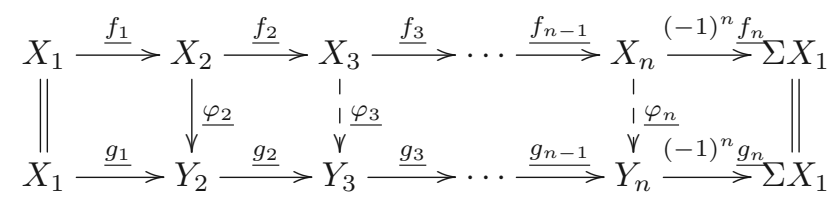

with rows standard $n$-angles, we need to show that there exist morphisms $\varphi_{i}: X_{i} \rightarrow Y_{i}$ for $3 \leqslant i \leqslant n$ such that $\underline{\varphi_{i} f_{i-1}}=\underline{g_{i-1} \varphi_{i-1}}$ and $\underline{f_{n}}=\underline{g_{n} \varphi_{n}}$, and the following

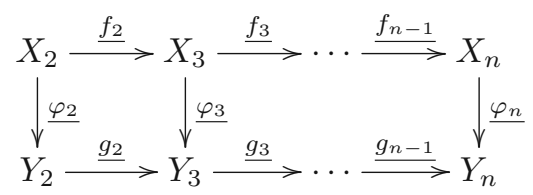

is a homotopy cartesian diagram whose differential is $(-1)^{n} \Sigma f_{1} \cdot \underline{g_{n}}$.

Indeed, we assume that

$$
0 \rightarrow X_{1} \stackrel{f_{1}}{\longrightarrow} X_{2} \stackrel{f_{2}}{\longrightarrow} X_{3} \stackrel{f_{3}}{\longrightarrow} \cdots \stackrel{f_{n-1}}{\longrightarrow} X_{n} \rightarrow 0
$$

and

$$
0 \rightarrow X_{1} \stackrel{g_{1}}{\longrightarrow} Y_{2} \stackrel{g_{2}}{\longrightarrow} Y_{3} \stackrel{g_{3}}{\longrightarrow} \cdots \stackrel{g_{n-1}}{\longrightarrow} Y_{n} \rightarrow 0
$$

are $(n-2)$-exact sequences in $\mathcal{X}$. Since $g_{1}=\varphi_{2} f_{1}$, there exist two morphisms $a: X_{1} \rightarrow I$ and $b: I \rightarrow Y_{2}$ such that $I \in \mathcal{I}$ and $\overline{g_{1}-} \varphi_{2} f_{1}=b a$. Since $I \in \mathcal{I}$, there is a morphism $c: X_{2} \rightarrow I$ such that $a=c f_{1}$. Thus, $g_{1}=\left(\varphi_{2}+b c\right) f_{1}$. Consequently, there exist morphisms $\varphi_{i}: X_{i} \rightarrow Y_{i}$ for $3 \leqslant i \leqslant n$ such that the following diagram

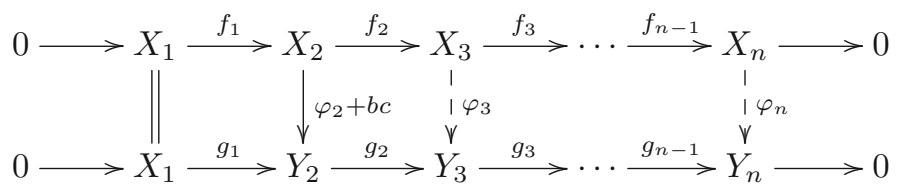

is commutative. Hence, the sequence

$0 \rightarrow X_{2} \stackrel{\left(\begin{array}{c}-f_{2} \\ \varphi_{2}+b c\end{array}\right)}{\longrightarrow} X_{3} \oplus Y_{2} \stackrel{\left(\begin{array}{cc}-f_{3} & 0 \\ \varphi_{3} & g_{2}\end{array}\right)}{\longrightarrow} \cdots \stackrel{\left(\begin{array}{cc}-f_{n-1} & 0 \\ \varphi_{n-1} & g_{n-2}\end{array}\right)}{\longrightarrow} X_{n} \oplus Y_{n-1} \stackrel{\left(\varphi_{n}, g_{n-1}\right)}{\longrightarrow} Y_{n} \rightarrow 0$

is an $(n-2)$-exact sequence in $\mathcal{X}$ by the dual of Lemma 4.3 , which induces the following standard $n$-angle

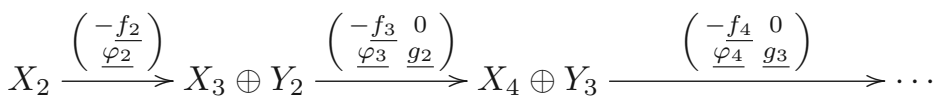

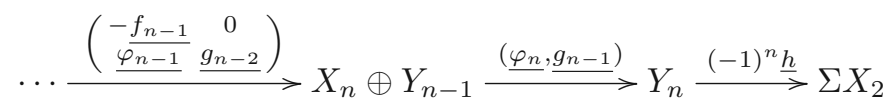


for some morphism $h: Y_{n} \rightarrow \Sigma X_{2}$. The following commutative diagram

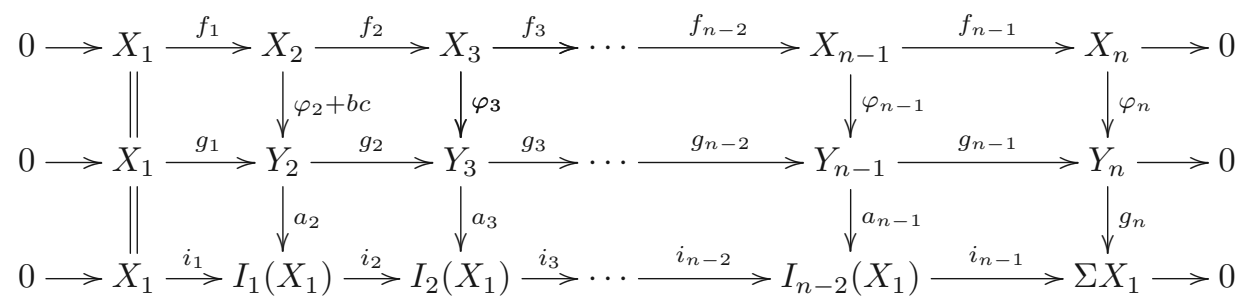

and Lemma 4.4 implies that $f_{n}=g_{n} \varphi_{n}$. Comparing the following two diagrams

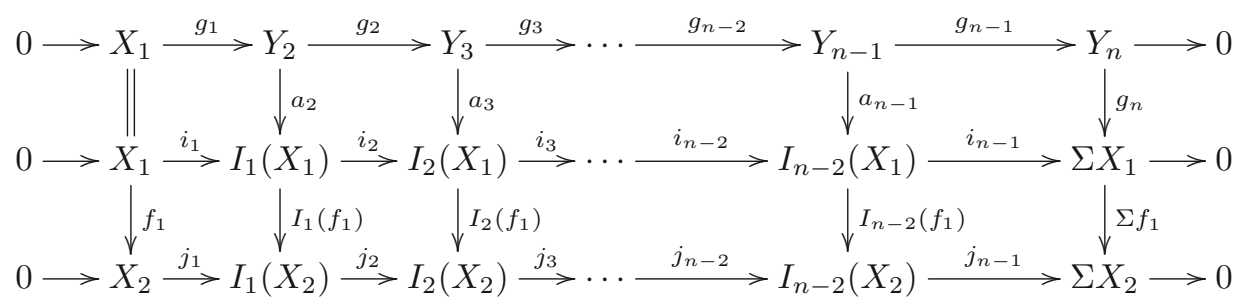

and

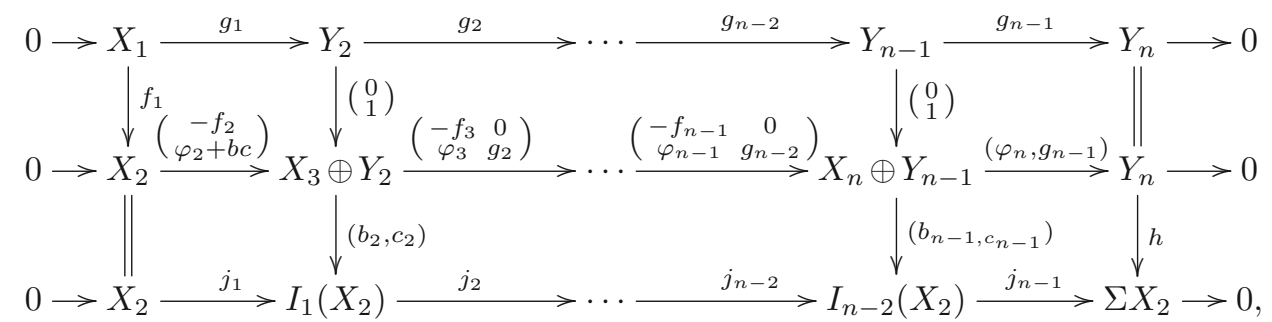

we have $\underline{h}=\Sigma \underline{f_{1}} \cdot \underline{g_{n}}$ by Lemma 4.4. We are done.

\section{Acknowledgments}

Some part of the work was done when the first author visited University of Stuttgart. He wants to thank Steffen Koenig for warm hospitality during his stay in Stuttgart. The authors sincerely thank the referee for his or her helpful comments and suggestions improving this paper. This work was partially supported by the Foundation of the Education Department of Fujian Province (Grant No. JZ160405) and Natural Science Foundation of China (Grant No. 11871014 and No. 11871259).

\section{References}

[1] E. Arentz-Hansen, P.A. Bergh, M. Thaule, The morphism axiom for $n$ angulated categories, Theory Appl. Categ., 31(18) (2016), 477-483.

[2] A. Beligiannis, Relative homological algebra and purity in triangulated Categories, J. Algebra, 227(2000), 268-361.

[3] P.A. Bergh, M. Thaule, The axioms for $n$-angulated categories, Algebr. Geom. Topol., 13(4) (2013), 2405-2428. 
[4] P.A. Bergh, G. Jasso, M. Thaule, Higher $n$-angulations from local rings, J. Lond. Math. Soc., 93(1) (2016), 123-142.

[5] C. Geiss, B. Keller, S. Oppermann, n-angulated categories, J. Reine Angew. Math., 675 (2013), 101-120.

[6] G. Jasso, n-abelian and n-exact categories, Math. Z., 283(3) (2016), 703-759.

[7] H. Krause, Derived categories, resolutions, and Brown representability, in: Interactions Between Homotopy Theory and Algebra, 101-139, in: Contemp. Math., 436, Amer. Math. Soc. Providence, RI, 2007.

[8] Z. Lin, A general construction of $n$-angulated categories using periodic injective resolutions, J. Pure Appl. Algebra, 223 (2019), 3129-3149.

[9] A. Neeman, Triangulated Categories, Ann. of Math. Stud., 148, Princeton University Press, Princeton, NJ, 2001.

[10] B.J. Parshall, L.L. Scott, Derived categories, quasi-hereditary algebras, and algebraic groups, Carleton Univ. Math. Notes, 3 (1988), 1-144.

Zengqiang Lin zqlin@hqu.edu.cn

School of Mathematical Sciences, Huaqiao University, No. 269 Chenghua North Road, Quanzhou, Fujian, 362021, China

Yan Zheng zy112291@163.com

School of Mathematical Sciences, Huaqiao University, No. 269 Chenghua North Road, Quanzhou, Fujian, 362021, China 